\title{
Estrategias no farmacológicas en el paciente clínicamente enfermo, ¿qué nos dice la literatura?
}

\section{Non-pharmacological Strategies in the Clinically Ill Patient, What does the Literature Tell Us?}

Fecha de recepción: 26 Abril 2016 | Fecha de aprobación: 06 Junio 2017

Julián Andrés Sucerquia Quintero a

Novartis, Colombia

a Autor de corresponencia. Correo electrónico: jsucer@gmail.com

Cómo citar: Sucerquia Quintero JA. Estrategias no farmacológicas en el paciente clínicamente enfermo: ¿qué nos dice la literatura? Univ Med. 2017; 58(3):114. doi: https://doi.org/10.11144/Javeriana.umed58-3. farm

\section{RESUMEN}

En el paciente médicamente enfermo, las intervenciones farmacológicas o psicoterapéuticas habituales caen en desuso por particularidades propias de la hospitalización y la comorbilidad física; por ello se hace necesario identificar diferentes estrategias que permitan incidir benéficamente en la calidad de vida de este grupo de pacientes. Objetivo: Realizar una revisión narrativa de la literatura que permita identificar estrategias terapéuticas que hayan demostrado efectividad en el proceso de este grupo de pacientes. Metodología: Revisión narrativa de literatura. Resultados: Se identificaron 10 corrientes terapéuticas no farmacológicas que han demostrado mejoría en indicadores como percepción de dolor, mejoría en calidad de vida, regulación de alteraciones del patrón de sueño, fatiga, en el contexto de pacientes con enfermedad crónica, que puede encontrarse en entornos tan diversos como son los pacientes de oncología o de las unidades de dolor crónico. Conclusiones: Las intervenciones no farmacológicas han demostrado efectividad en el control de síntomas físicos y emocionales en pacientes con enfermedad crónica y son herramientas con las que debe contar el profesional en salud mental.

\section{Palabras clave}

relajación; cáncer; dolor crónico.

\begin{abstract}
For the treatment of the clinically ill patient the usual pharmacological interventions have limited the use of non-pharmacological approach, due to particular factors such as hospitalization itself, physical comorbidities or reduced strategies for the therapeutic team; it is necessary to identify therapeutical strategies that allow a positive effect in the quality of life of this group of patients. Objective: To realize a narrative review of literature that allows us to identify therapeutically strategies that have proven electivity in the clinically ill patient. Methods: Narrative review of literature. Results: There have been identified 10 non pharmacological therapeutic options that have demonstrated a positive effect in domains such as pain perception, quality of life perception, insomnia, fatigue, in the context of chronic and clinically ill patients in services such as oncology or chronic pain. Conclusions: Non-pharmacological interventions have proven effectivity in ameliorate physical and emotional symptoms in patients with chronic diseases, and are useful tools for the mental health specialist.
\end{abstract}


Keywords

relaxation; cancer; chronic pain.

\section{Introducción}

El trabajo del especialista en psiquiatría, al trabajar en el hospital general, se ve enfrentado a las complejidades del paciente clínicamente enfermo en situaciones tan complejas como son la unidad de oncología o las unidades de dolor crónico, en quienes las intervenciones farmacológicas "usuales" no presentan resultados completos, al indagar acerca de la mejoría en su calidad de vida. Estas situaciones pueden ser favorecidas por las complejidades propias del escenario asistencial, determinadas por factores ambientales, afectación físico y mental asociada a la patología médica, tiempo destinado para la intervención, otros procedimientos que debe realizarse el paciente, etcétera. De ahí surge la necesidad para el profesional de psiquiatría de enlace de aplicar otras alternativas y de diversificar así el arsenal terapéutico con el que cuenta como profesional de la salud, al enfrentarse al paciente clínicamente enfermo [1], en escenarios como el trabajo interdisciplinario en los diferentes servicios de interconsultas y enlace, dado que dentro de su labor debe identificar e implementar estrategias terapéuticas que tengan un beneficio real demostrable en la calidad de vida del individuo [2].

Al trabajar en ambientes clínicos complejos, por ejemplo aquellos en los que se encuentra el paciente con requerimiento de hospitalización prolongada o enfermedad crónica, como puede ser una situación cotidiana en los servicios de medicina interna $[3,4,5,6]$, oncología $[2,7,8,9,10]$, unidades de rehabilitación funcional y otros donde es una necesidad manifiesta el identificar estrategias terapéuticas diferentes a las farmacológicas, encaminadas a actuar positivamente en el estado mental de ese individuo que requiere satisfacción global de sus necesidades, en un enfoque integral $[11,12,13]$, que garantiza también el de sus familiares [14, 15]. Si bien es cierto que existe el recurso de los psicofármacos, con el cual está ampliamente familiarizado todo profesional de la salud y su empleo es bastante cotidiano, en la literatura se ha planteado que para síntomas de intensidad leve a moderada en trastornos tanto ansiosos como del afecto, la herramienta principal sería una intervención no farmacológica [16, 17, 18, $19,20,21,22]$.

También es claro que en el momento de proponer estrategias no farmacológicas, nos encontramos ante la inquietud de si dicho tipo de intervenciones cuenta con un respaldo basado en ensayos clínicos y evidencia científica suficiente, que permita argumentar en el escenario del trabajo diario un sustento de las bases sobre las cuales se plantea. Esta particularidad puede generar moderada discusión en el interior de equipo interdisciplinar, donde es importante brindar siempre estrategias desde una perspectiva crítica y sustentada en la evidencia científica.

\section{Objetivo}

Es objeto de este artículo realizar una búsqueda narrativa de información actualizada en revistas indexadas en la base de datos Medline, a fin de brindar herramientas al profesional de la salud mental en el momento de argumentar la existencia, la importancia y la efectividad de estrategias terapéuticas diferentes a las farmacológicas, en escritos fundamentados en literatura científica, pues es de conocimiento general que se favorecen estrategias mediadas en agentes químicos que pueden acarrear posibles efectos no deseados en el contexto de un paciente, cuya estabilidad orgánica se ve afectada por diversas complicaciones propias de un proceso crónico de enfermedad. No es el fin de este artículo determinar una única corriente u opción terapéutica, sino sentar claridad respecto a diferentes estrategias que han brindado evidencia en su efectividad.

\section{Metodología}

Se llevó a cabo una revisión narrativa de la literatura, empleando la base de datos Medline. Se buscaron los términos MeSH terminal care, realizando cruce con los términos, mind-body 
therapies, cognitive therapy, recreation therapy, psychoanalytic therapy, behavior therapy, imagery (psychotherapy), narrative therapy, family therapy OR psychotherapy, brief. Se excluyó también en la búsqueda inicial aquellas referencias que incluyeran el término drug therapy.

Con la primera búsqueda se encontraron 72.818 referencias bibliográficas. Luego se eligieron aquellas que constituyeran ensayos clínicos (clinical trials), para una posterior reducción a 11.003 referencias. Se decidió agrupar los artículos que se encontraban bajo la categoría cáncer y se lograron 1873 referencias. Entonces se limitó por aquellos estudios que se centraban en población adulta (mayor 19 años), de lo cual se obtuvieron 1538 resultados. El último filtro correspondió a los artículos que se hubieran publicado en los últimos cinco años, que produjo una reducción a 509 artículos. Se leyeron los resúmenes de este grupo, del cual se escogieron 136 artículos, ya que cumplían con las condiciones de la búsqueda inicial, pues otros se centraban en enfermedades mentales crónicas, como trastornos psicóticos, del afecto o ansiosos, o hacían referencia a población pediátrica, lo que no corresponde con los objetivos del presente trabajo. El proceso de selección queda reflejado en la figura 1.
Figura 1.Proceso de selección de artículos para el estudio

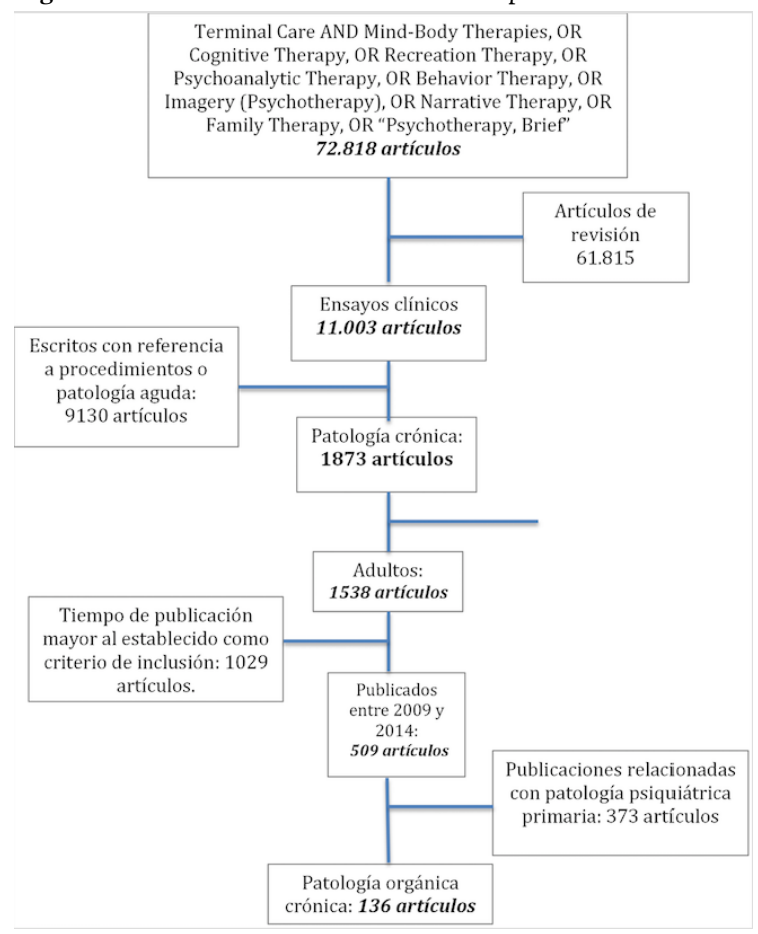

Es necesario aclarar que, a pesar de los criterios de selección, algunos de los artículos se relacionaban con condiciones crónicas de patología no oncológica, entre las cuales se encuentran la fibromialgia [23], el VIH [24, 25], el lupus eritematoso sistémico [26], la dermatitis atópica [27], secuelas de enfermedad cerebrovascular [28, 29], el dolor crónico de espalda y cuello $[6,30,31,32]$, así como la fatiga crónica [33]. Sin embargo, de los 136 artículos referenciados, el $69 \%$ (n = 94) correspondía a intervenciones realizadas con población oncológica, entre los cuales las poblaciones descritas son cáncer de seno y sus complicaciones en mujeres (48\%) [34, $35]$, y sobrevivientes de cáncer de próstata en hombres (6,4\%) [36]; mientras que el excedente correspondía a las demás patologías crónicas que afectan la percepción de calidad de vida de los pacientes, no relacionadas a patología oncológica [11].

Una vez seleccionados los artículos para el presente trabajo, se identificaron los elementos de intervención no farmacológica que eran sujetos de descripción: 30 artículos hacen 
referencia al proceso psicoterapéutico conocido como mindfulness; 51, a la terapia cognitivacomportamental; 15, a la aplicación de técnicas de yoga; mientras que 17 respecto a otras técnicas de relajación. Únicamente 2 de los artículos eran sobre la aplicación de técnicas de hipnosis y otros 16 se referían a modificaciones en el estilo de vida. Dos de los artículos explicitan técnicas de psicoterapia breve. En lo relacionado con terapia de grupo se identificaron 11 textos; mientras que a la aplicación de técnicas de musicoterapia se asocia con 5 de los artículos. Por lo demás, se encontraron otras psicoterapias, las cuales se describen más adelante en el texto. Lo anterior se encuentra explicitado en la figura 2.

Figura 2.Distribución de artículos por estrategia terapéutica

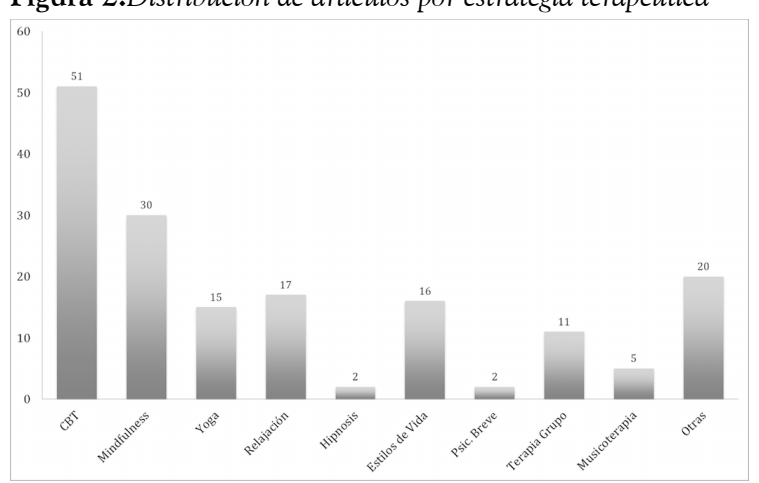

\section{Resultados}

Posterior a la revisión de los artículos, se identificó una medición del efecto esperado en varios grupos de síntomas, principalmente para buscar indicadores que permitieran cuantificar dicho impacto, generalmente centrándose en cuestionarios de autorregistro validados, como es el caso de la Escala de Autorreporte de Hamilton para Medir Ansiedad, la Escala de Zung para Depresión, el Cuestionario de Calidad de Vida (QOL), entre otros instrumentos. Se buscó generar categorías que permitieran identificar cada una de estas estrategias no farmacológicas y cómo impactan en la vida de las personas que las reciben dentro del arsenal terapéutico.

Al leer los diferentes artículos, se organizaron como características emergentes una serie de resultados categorizados como comunes y así se consiguió codificar el proceso a través del cual identificar los efectos de las diferentes intervenciones, los cuales se resumen a continuación:

Disminución de síntomas de ansiedad (72,1 $\%)$.

Mejoría en percepción de calidad de sueño/ disminución de insomnio (8,8 \%).

Disminución de la fatiga física $(27,2 \%)$.

Mejoría en adherencia a estrategias de tratamiento $(8,8 \%)$.

Disminución en la percepción de dolor (25\%).

Mejoría global en la percepción de calidad de vida $(77,2 \%)$.

Disminución de síntomas depresivos (16,9\%).

La distribución de artículos relacionados con cada una de las temáticas se muestra en la figura 3.

Figura 3.Distribución de artículos según categoría emergente

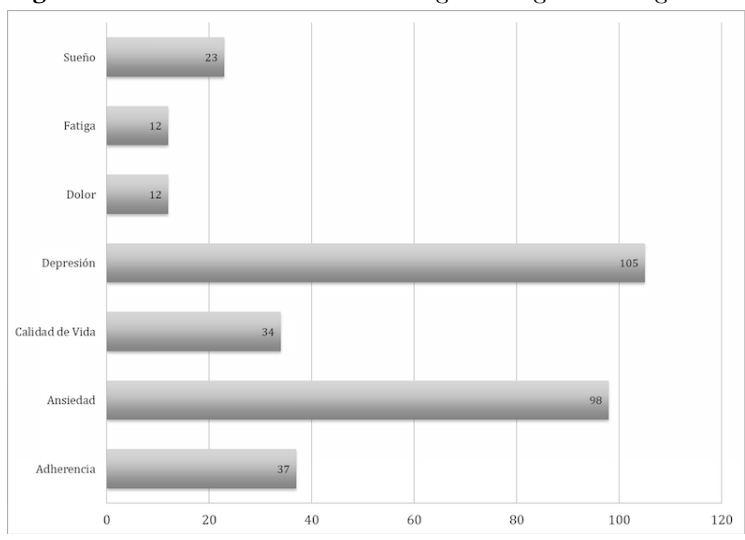

En las próximas líneas se reseñan brevemente los diferentes subtipos de estrategias terapéuticas no farmacológicas identificadas.

Terapia cognitivo-comportamental (30,2\%)

Generalmente administrada por un terapeuta, pero en algunas oportunidades por profesionales del área de enfermería [37] o, incluso, por medios distantes como una llamada telefónica [11]. La terapia cognitivo-comportamental consiste en estrategias de desensibilización sistemática y exposición progresiva que buscan identificar y detener los pensamientos automáticos negativos y los síntomas de ansiedad que se asocian. Se 
administra tanto para cáncer $[2,10,36,38,39$, 40, 41, 42, 43, 44, 45, 46, 47, 48, 49] como para otras patologías crónicas $[1,17,23,24,30,31$, 32, 50].

Los desenlaces considerados satisfactorios se concentran en identificar en los pacientes una disminución de la percepción de dolor y de la ansiedad, al igual que en controlar las alteraciones del patrón de sueño, lo cual se contempla tanto para patología tumoral como para la no tumoral. Ha sido comparada con modificaciones de estilo de vida y con otras estrategias terapéuticas. En conclusión, no hay una asociación estadísticamente significativa con un resultado catalogable como superior a otras intervenciones no farmacológicas; sin embargo, sí evidencian mejores resultados que en aquellas en las cuales no se hace intervención diferente a la medicación.

\section{Mindfulness $(17,8 \%)$}

Es una técnica terapéutica con variaciones del modelo cognitivo-comportamental, en la cual se procura "atención plena" al momento en el que se encuentra "con interés, curiosidad y aceptación". En los artículos seleccionados se halló el mindfulness con aplicación a la patología oncológica $[7,51,52,53,54,55,56,57$, 58, 59, 60, 61, 62, 63], así como su uso en otras patologías crónicas $[4,18,64,65]$. Se ha demostrado su efecto fisiológico en la reducción de marcadores de ansiedad, como la respuesta humana al cortisol $[66,67]$.

El mindfulness se ha comparado con la terapia cognitiva-comportamental $[16,68]$ para determinar cuál tiene una mayor efectividad en el contexto de pacientes oncológicos; por ello se han medido las características de percepción de dolor, ansiedad y control de alteraciones del patrón de sueño; siendo satisfactorios los resultados de ambas intervenciones. Sin embargo, no ha sido posible demostrar que tenga mayor efectividad que las estrategias de la terapia cognitiva-comportamental y se ha dejado en consideración la necesidad de implementar diseños diferentes de investigación para lograr esclarecer este último punto.

Psicoterapia breve (1,2\%)

La psicoterapia breve se entiende no como un modelo psicoterapéutico de una corriente establecida, sino que se cuantifica por el número de sesiones establecido. Se denomina breve, dado que su periodo es menor al establecido para el modelo de orientación cognitivo-comportamental, que oscila entre 12 y 16 sesiones $[5,20,38,69,70,71]$. Esta psicoterapia no se ha contrastado con otras estrategias terapéuticas, pero sí ha demostrado un efecto positivo en mejoría de calidad de vida y percepción de salud tanto en los pacientes como en sus familiares. Dentro de las metodologías descritas en los artículos relacionados, se aplicaban intervenciones centradas en optimizar tanto las estrategias de afrontamiento propias del individuo como la contención de la respuesta emocional en su núcleo familiar.

El subtipo de psicoterapia breve más utilizada, derivada del proceso psicoanalítico, hace referencia a la terapia psicodinámicamente orientada $[71,72,73,74,75,76,77,78,79$, $80,81,82,83,84,85,86,87,88,89,90$, 91, 92, 93, 94, 95, 96], y en uno de los artículos se plantea la posibilidad de diseñar un estudio que compare este tipo de intervenciones con otros modelos psicoterapéuticos [96]. Si bien no se menciona el número mínimo de sesiones necesario para garantizar una adecuada intervención, se recomienda que, como todo proceso psicoterapéutico, se centre en el individuo que se beneficiaría de ella, más que en intentar plantear una fórmula o algoritmo para reproducirlo de manera autónoma.

Modificaciones al estilo de vida (9,5\%)

Para este tipo de estrategias se utilizan pasos comunes a la psicoterapia breve, pero propiamente relacionada con el modelo de entrevista motivacional. Se busca incidir tanto en la reducción de factores de riesgo de patología 
oncológica como en el incremento de la actividad física, que se considera un factor optimizador de la percepción de salud del individuo, así como de regulación de alteraciones del patrón sueño (higiene) y disminución progresiva de la percepción de dolor, relacionado con cambios en los hábitos cotidianos [12, 72, 73, 74, 75]. No se establece per se un modelo psicoterapéutico, y el estilo empleado corresponde más a la confrontación empática que a otros aspectos propios del modelo conductual.

\section{Relajación (10,1\%)}

Esta estrategia propone técnicas de relajación muscular progresiva, al igual que biofeedback, que en ocasiones se asocia con elementos de aromaterapia y ejercicios de imaginería. Se propone como una alternativa a los analgésicos y a la medicación ansiolítica e hipnótica para el tratamiento de complicaciones del paciente oncológico o con dolor crónico. Como ventaja, está el hecho de no requerir largas sesiones de entrenamiento, y con la adición de una grabación para recordar los pasos, gracias a los cuales se alcanza el estado de relajación. No requiere visitas seriadas al terapeuta $[13,22,27,76,77$, $78,79,80,81]$.

\section{Yoga $(8,9 \%)$}

El yoga se comprende como ejercicios dirigidos, centrados en la respiración y la relajación muscular progresiva, bajo la tutela de un terapeuta experimentado. Se ha demostrado su efectividad en el control de la respiración asociada a patología tumoral, así como atenuación en síntomas ansiosos y disminución en la percepción de dolor de diferentes enfermedades crónicas. De igual forma, es una estrategia benéfica para la regulación de alteraciones del patrón de sueño, pero no se ha evaluado comparativamente con otras estrategias terapéuticas. En los ensayos clínicos controlados tiene buen impacto en la percepción de calidad de vida tanto del paciente como de su grupo familiar [82, 83, 84, 85, 86, 87, 88].

\section{Terapia de grupo $(6,5 \%)$}

Independiente del modelo terapéutico con que se haga el encuadre, los grupos de terapia han demostrado un efecto positivo en la percepción de calidad de vida, así como en la perpetuación de modificaciones en el estilo de vida, que permiten atenuar las limitaciones propias de la enfermedad crónica. En los artículos obtenidos para esta revisión, su empleo se ha descrito tanto para patología oncológica como para otras dolencias crónicas, con efectos benéficos dignos de resaltar. Se han descrito el modelo cognitivocomportamental y las técnicas de respiración/ relajación grupales, incluso la terapia dialéctica e interpersonal [6, 34, 45, 89, 90].

\section{Musicoterapia (3\%)}

Entendida como la utilización de sonidos armoniosos para estimulación sensorial y la distracción del foco de atención, cuando este se ve centrado en experiencias displacenteras o dolorosas, como suele ocurrir con los pacientes con patología oncológica. Sin embargo, la aplicación de la musicoterapia no solo se limita al tratamiento de enfermedades tumorales, sino que se ha asociado con la percepción de mejoría en calidad de vida de patologías crónicas como la fibromialgia. No se ha demostrado que un tipo de música sea superior a los demás para tal fin; los distintos ensayos coinciden en afirmar que lo importante es que el individuo encuentre la experiencia y el estímulo como placenteros [91, 92, 93, 94].

\section{Hipnosis (1,2\%)}

Esta modalidad terapéutica, si bien ha demostrado efectividad en el control y disminución de la percepción de dolor, así como en la regulación del patrón de sueño y mejoría global en indicadores de calidad de vida, no se ha empleado de manera generalizada, por la dificultad para garantizar métodos uniformes de estandarizar sus resultados y proceso. Sigue 
siendo una herramienta útil en la patología oncológica para el tratamiento de síntomas como las náuseas y la percepción de dolor, así como en patologías dolorosas no tumorales. Requiere un adecuado entrenamiento por parte del terapeuta, razón por la que no se convierte en la primera estrategia empleada por los profesionales de la salud $[44,97]$.

Otras psicoterapias $(11,8 \%)$

Al generar esta categoría, se encontraron artículos con técnicas tan diversas como la terapia emotiva-comportamental, la acupresión [78], la acupuntura [79], la terapia existencialcomportamental, la reflexología y aromaterapia [72], la terapia asistida telefónicamente, las estrategias relacionadas con software, las terapias narrativas, la terapia relacionada con imágenes e interpretación [98], entre otras tantas. Se han descrito para cada una por separado atenuación de síntomas ansiosos y síntomas depresivos o mejoría en el patrón de sueño y en la percepción general de calidad de vida; sin embargo, en un número no suficiente para agruparse entre ellas.

\section{Discusión}

En la práctica clínica se evidencia la necesidad de contar con estrategias terapéuticas que optimicen la percepción de calidad de vida, y esto es muy importante en el paciente clínicamente enfermo con patología crónica asociada a percepción de dolor o con interferencia en su funcionalidad. Es potestad del profesional en salud mental, y en este caso del especialista en psiquiatría de enlace, conocer los beneficios de diferentes tipos de modelos terapéuticos no farmacológicos; así como poder argumentar de una manera racional y con rigor científico su implementación en el contexto del paciente médicamente enfermo.

$\mathrm{Si}$ bien es cierto que la mayoría de estudios hace referencia a estrategias del estilo cognitivo-comportamental, así como al modelo de atención plena (mindfulness), también queda evidenciado que otros modelos terapéuticos como relajación, ejercicios de respiración, aplicación de técnicas de yoga, psicoterapia breve e hipnosis tienen utilidad; sin embargo, es necesaria la comparación directa entre estas diversas estrategias terapéuticas, a fin de identificar modelos de mayor efectividad.

Al identificar las estrategias no farmacológicas empleadas en los diferentes ensayos clínicos, se aclara que son de fácil implementación, dado que no precisan recursos especializados ni tecnología de punta, sino un equipo conformado por profesionales con experiencia en las diferentes áreas. Al realizar una intervención cualquiera en un paciente, se deben considerar los fenómenos de riesgo y beneficio, lo cual permite practicar estrategias en las cuales se procuran intervenciones no invasivas, que atenúen los síntomas cognitivos y emocionales, que pueden exponer a los pacientes a percibir como una amenaza los síntomas físicos propios de enfermedades crónicas, tanto oncológicas como no oncológicas.

\section{Conclusiones}

El campo de las intervenciones terapéuticas en unidades de pacientes con enfermedad crónica es una necesidad manifiesta por parte de los usuarios del sistema de salud; así como una oportunidad para recuperar esos espacios que la psiquiatría, como especialidad médica, ha perdido al concentrarse en la consulta privada y en las unidades de salud mental.

Entre las limitaciones de esta revisión se encuentra que no hay una medida estandarizada para identificar el impacto de cada una de las intervenciones, en los diferentes estudios. Esto quiere decir que se hace una comparación de acuerdo con lo identificado con las herramientas de seguimiento de cada estudio (escalas de autorreporte, entrevista semiestructurada, etc.).

Otra limitación corresponde a la no identificación de estudios que permitan establecer una comparación directa entre diferentes tipos de intervención no farmacológica. 
En el momento de llevar a cabo cualquier tipo de intervención terapéutica en un paciente, es necesario sopesar sus riesgos y beneficios; así como la costoefectividad de dicha estrategia. Las estrategias terapéuticas no farmacológicas no precisan recursos de última tecnología, lo cual las convierte en herramientas viables de bajo costo económico, con mejoría en la percepción global de calidad de vida de los pacientes que se benefician de ellas.

\section{Agradecimientos}

Al doctor Carlos Gómez-Restrepo, psiquiatra y epidemiólogo, por su actividad como docente en el programa de Especialización de Psiquiatría de Enlace y la motivación a la lectura crítica. A la doctora Sandra Milena Ramírez Rivera, psiquiatra de enlace, por su paciencia y motivación en el proceso de búsqueda y redacción de este documento.

\section{Referencias}

1. Samwel HJ, Kraaimaat FW, Crul BJ, van Dongen RD, Evers AW. Multidisciplinary allocation of chronic pain treatment: effects and cognitive-behavioural predictors of outcome. Br J Health Psychol. 2009;14(Pt 3):405-21.

2. Antoni $\mathrm{MH}$, Lechner S, Diaz A, Vargas S, Holley H, Phillips K, et al. Cognitive behavioral stress management effects on psychosocial and physiological adaptation in women undergoing treatment for breast cancer. Brain Behav Immun. 2009;23(5):580-91.

3. Mahigir F, Khanehkeshi A, Karimi A. Psychological treatment for pain among cancer patients by rational-emotive behavior therapy--efficacy in both India and Iran. Asian Pac J Cancer Prev. 2012;13(9):4561-5.

4. Rosenkranz MA, Davidson RJ, Maccoon DG, Sheridan JF, Kalin NH, Lutz A. A comparison of mindfulness-based stress reduction and an active control in modulation of neurogenic inflammation. Brain Behav Immun. 2013;27 (1):174-84.

5. Wu DY, Guo M, Gao YS, Kang YH, Guo JC, Jiang XL, et al. Clinical effects of psychological intervention and drug therapy against peptic ulcer. Asian Pac J Trop Med. 2012;5(10):831-3.

6. Dinkel A, Herschbach P, Berg P, Waadt S, Duran G, Engst-Hastreiter U, et al. Determinants of long-term response to group therapy for dysfunctional fear of progression in chronic diseases. Behav Med (Washington, DC). 2012;38(1):1-5.

7. Andersen SR, Wurtzen H, Steding-Jessen M, Christensen J, Andersen KK, Flyger H, et al. Effect of mindfulness-based stress reduction on sleep quality: results of a randomized trial among Danish breast cancer patients. Acta Oncol. 2013;52(2):336-44.

8. Boesen EH, Karlsen R, Christensen J, Paaschburg B, Nielsen D, Bloch IS, et al. Psychosocial group intervention for patients with primary breast cancer: a randomised trial. Eur J Cancer. 2011;47(9):1363-72.

9. Heinrichs N, Zimmermann T, Huber B, Herschbach P, Russell DW, Baucom DH. Cancer distress reduction with a couple-based skills training: a randomized controlled trial. Ann Behav Med. 2012;43(2):239-52.

10. Kesler S, Hadi Hosseini SM, Heckler C, Janelsins M, Palesh O, Mustian K, et al. Cognitive training for improving executive function in chemotherapy-treated breast cancer survivors. Clin Breast Cancer. 2013;13(4):299-306.

11. DuHamel KN, Mosher CE, Winkel G, Labay LE, Rini C, Meschian YM, et al. Randomized clinical trial of telephoneadministered cognitive-behavioral therapy to reduce post-traumatic stress disorder and distress symptoms after hematopoietic stem-cell transplantation. J Clin Oncol. 2010;28(23):3754-61.

12. Gaston-Johansson F, Fall-Dickson JM, Nanda JP, Sarenmalm EK, Browall M, Goldstein $\mathrm{N}$. Long-term effect of the self-management 
comprehensive coping strategy program on quality of life in patients with breast cancer treated with high-dose chemotherapy. Psycho-oncology. 2013;22(3):530-9.

13. Park ER, Traeger L, Willett J, Gerade B, Webster A, Rastegar S, et al. A relaxation response training for women undergoing breast biopsy: exploring integrated care. Breast. 2013;22(5):799-805.

14. Northouse LL, Mood DW, Schafenacker A, Kalemkerian G, Zalupski M, LoRusso P, et al. Randomized clinical trial of a brief and extensive dyadic intervention for advanced cancer patients and their family caregivers. Psycho-oncology. 2013;22(3):555-63.

15. Iobst EA, Alderfer MA, Sahler OJ, Askins MA, Fairclough DL, Katz ER, et al. Problem solving and maternal distress at the time of a child's diagnosis of cancer in two-parent versus lone-parent households. J Pediatr Psychol. 2009;34(8):817-21.

16. Garland SN, Carlson LE, Stephens AJ, Antle MC, Samuels C, Campbell TS. Mindfulness-based stress reduction compared with cognitive behavioral therapy for the treatment of insomnia comorbid with cancer: a randomized, partially blinded, noninferiority trial. J Clin Oncol. 2014;32(5):449-57.

17. Vitiello MV, Rybarczyk B, Von Korff M, Stepanski EJ. Cognitive behavioral therapy for insomnia improves sleep and decreases pain in older adults with co-morbid insomnia and osteoarthritis. J Clin Sleep Med. 2009;5(4):355-62.

18. Benzo RP. Mindfulness and motivational interviewing: two candidate methods for promoting self-management. Chron Respir Dis. 2013;10(3):175-82.

19. Wells AA, Palinkas LA, Shon EJ, Ell K. Low-income cancer patients in depression treatment: dropouts and completers. J Behav Health Serv Res. 2013;40(4):427-41.

20. Blais MA, Malone JC, Stein MB, Slavin-Mulford J, O'Keefe SM, Renna $M$, et al. Treatment as usual (TAU) for depression: a comparison of psychotherapy, pharmacotherapy, and combined treatment at a large academic medical center. Psychotherapy (Chicago, Ill). 2013;50(1):110-8.

21. Lam RW, Parikh SV, Ramasubbu R, Michalak EE, Tam EM, Axler A, et al. Effects of combined pharmacotherapy and psychotherapy for improving work functioning in major depressive disorder. $\mathrm{Br}$ J Psychiatry. 2013;203(5):358-65.

22. Jane SW, Chen SL, Wilkie DJ, Lin YC, Foreman SW, Beaton RD, et al. Effects of massage on pain, mood status, relaxation, and sleep in Taiwanese patients with metastatic bone pain: a randomized clinical trial. Pain. 2011;152(10):2432-42.

23. van Koulil S, van Lankveld W, Kraaimaat FW, van Helmond T, Vedder A, van Hoorn $\mathrm{H}$, et al. Tailored cognitive-behavioural therapy and exercise training improves the physical fitness of patients with fibromyalgia. Ann Rheum Dis. 2011;70(12):2131-3.

24. Jensen SE, Pereira DB, Whitehead N, Buscher I, McCalla J, Andrasik M, et al. Cognitive-behavioral stress management and psychological well-being in HIV + racial/ethnic minority women with human papillomavirus. Health Psychol. 2013;32(2):227-30.

25. SeyedAlinaghi S, Jam S, Foroughi M, Imani A, Mohraz M, Djavid GE, et al. Randomized controlled trial of mindfulness-based stress reduction delivered to human immunodeficiency virus-positive patients in Iran: effects on CD4(+) T lymphocyte count and medical and psychological symptoms. Psychosom Med. 2012;74(6):620-7.

26. Navarrete-Navarrete N, Peralta-Ramirez MI, Sabio-Sanchez JM, Coin MA, RoblesOrtega H, Hidalgo-Tenorio C, et al. Efficacy of cognitive behavioural therapy for the treatment of chronic stress in patients with lupus erythematosus: a randomized controlled trial. Psychother Psychosom. 2010;79(2):107-15.

27. Bae BG, Oh SH, Park CO, Noh S, Noh JY, Kim KR, et al. Progressive muscle 
relaxation therapy for atopic dermatitis: objective assessment of efficacy. Acta Derm Venereol. 2012;92(1):57-61.

28. Basta D, Rossi-Izquierdo M, Soto-Varela A, Greters ME, Bittar RS, SteinhagenThiessen E, et al. Efficacy of a vibrotactile neurofeedback training in stance and gait conditions for the treatment of balance deficits: a double-blind, placebo-controlled multicenter study. Otol Neurotol. 2011;32(9):1492-9.

29. Druzbicki M, Kwolek A, Depa A, Przysada G. The use of a treadmill with biofeedback function in assessment of relearning walking skills in post-stroke hemiplegic patients--a preliminary report. Neurol Neurochirur Pol. 2010;44(6):567-73.

30. Andersson G, Johansson C, Nordlander A, Asmundson GJ. Chronic pain in older adults: a controlled pilot trial of a brief cognitive-behavioural group treatment. Behav Cogn Psychother. 2012;40(2):239-44.

31. Monticone M, Baiardi P, Vanti C, Ferrari S, Nava T, Montironi C, et al. Chronic neck pain and treatment of cognitive and behavioural factors: results of a randomised controlled clinical trial. Eur Spine J. 2012;21(8):1558-66.

32. McBeth J, Prescott G, Scotland G, Lovell K, Keeley P, Hannaford P, et al. Cognitive behavior therapy, exercise, or both for treating chronic widespread pain. Arch Intern Med. 2012;172(1):48-57.

33. Schreurs KM, Veehof MM, Passade L, Vollenbroek-Hutten MM. Cognitive behavioural treatment for chronic fatigue syndrome in a rehabilitation setting: effectiveness and predictors of outcome. Behav Res Ther. 2011;49(12):908-13.

34. Jones JM, Cheng T, Jackman M, Walton T, Haines S, Rodin G, et al. Getting back on track: evaluation of a brief group psychoeducation intervention for women completing primary treatment for breast cancer. Psycho-oncology. 2013;22(1):117-24.
35. Henderson VP, Clemow L, Massion AO, Hurley TG, Druker S, Hebert JR. The effects of mindfulness-based stress reduction on psychosocial outcomes and quality of life in early-stage breast cancer patients: a randomized trial. Breast Cancer Res Treat. 2012;131(1):99-109.

36. Traeger L, Penedo FJ, Benedict C, Dahn JR, Lechner SC, Schneiderman N, et al. Identifying how and for whom cognitive-behavioral stress management improves emotional well-being among recent prostate cancer survivors. Psychooncology. 2013;22(2):250-9.

37. Lee H, Lim Y, Yoo MS, Kim Y. Effects of a nurse-led cognitive-behavior therapy on fatigue and quality of life of patients with breast cancer undergoing radiotherapy: an exploratory study. Cancer Nurs. 2011;34(6):E22-30.

38. Cherrier MM, Anderson K, David D, Higano CS, Gray H, Church A, et al. A randomized trial of cognitive rehabilitation in cancer survivors. Life Sci. 2013;93(17):617-22.

39. Choi J, Kuo CW, Sikorskii A, You M, Ren D, Sherwood PR, et al. Cognitive behavioral symptom management intervention in patients with cancer: survival analysis. Support Care Cancer. 2012;20(6):1243-50.

40. Duijts SF, van Beurden M, Oldenburg HS, Hunter MS, Kieffer JM, Stuiver MM, et al. Efficacy of cognitive behavioral therapy and physical exercise in alleviating treatmentinduced menopausal symptoms in patients with breast cancer: results of a randomized, controlled, multicenter trial. J Clin Oncol. 2012;30(33):4124-33.

41. Groarke A, Curtis R, Kerin M. Cognitivebehavioural stress management enhances adjustment in women with breast cancer. $\mathrm{Br}$ J Health Psychol. 2013;18(3):623-41.

42. Korstjens I, Mesters I, May AM, van Weert E, van den Hout JH, Ros W, et al. Effects of cancer rehabilitation on problem-solving, anxiety and depression: a RCT comparing physical and cognitive-behavioural training versus physical training. Psychol Health. 2011;26 Suppl 1:63-82. 
43. McKiernan A, Steggles S, Guerin S, Carr A. A controlled trial of group cognitive behavior therapy for Irish breast cancer patients. J Psychosoc Oncol. 2010;28(2):143-56.

44. Montgomery GH, David D, Kangas M, Green S, Sucala M, Bovbjerg DH, et al. Randomized controlled trial of a cognitive-behavioral therapy plus hypnosis intervention to control fatigue in patients undergoing radiotherapy for breast cancer. J Clin Oncol. 2014;32(6):557-63.

45. Sabariego C, Brach M, Herschbach P, Berg P, Stucki G. Cost-effectiveness of cognitivebehavioral group therapy for dysfunctional fear of progression in cancer patients. Eur J Health Econ. 2011;12(5):489-97.

46. Sansom-Daly UM, Wakefield CE, Bryant RA, Butow P, Sawyer S, Patterson P, et al. Online group-based cognitive-behavioural therapy for adolescents and young adults after cancer treatment: a multicenter randomised controlled trial of Recapture Life-AYA. BMC cancer. 2012;12:339.

47. Tremblay V, Savard J, Ivers H. Predictors of the effect of cognitive behavioral therapy for chronic insomnia comorbid with breast cancer. J Consult Clin Psychol. 2009;77 (4):742-50.

48. van Weert E, May AM, Korstjens I, Post WJ, van der Schans CP, van den Borne B, et al. Cancer-related fatigue and rehabilitation: a randomized controlled multicenter trial comparing physical training combined with cognitive-behavioral therapy with physical training only and with no intervention. Phys Ther. 2010;90(10):1413-25.

49. Von Ah D, Carpenter JS, Saykin A, Monahan P, Wu J, Yu M, et al. Advanced cognitive training for breast cancer survivors: a randomized controlled trial. Breast Cancer Res Treat. 2012;135(3):799-809.

50. Glombiewski JA, Hartwich-Tersek J, Rief W. Two psychological interventions are effective in severely disabled, chronic back pain patients: a randomised controlled trial. Int J Behav Med. 2010;17(2):97-107.
51. Ando $\mathrm{M}$, Morita $\mathrm{T}$, Akechi $\mathrm{T}$, Ito $\mathrm{S}$, Tanaka M, Ifuku Y, et al. The efficacy of mindfulness-based meditation therapy on anxiety, depression, and spirituality in Japanese patients with cancer. J Palliat Med. 2009;12(12):1091-4.

52. Branstrom R, Kvillemo P, Brandberg Y, Moskowitz JT. Self-report mindfulness as a mediator of psychological well-being in a stress reduction intervention for cancer patients--a randomized study. Ann Behav Med. 2010;39(2):151-61.

53. Chambers SK, Smith DP, Berry M, Lepore SJ, Foley E, Clutton S, et al. A randomised controlled trial of a mindfulness intervention for men with advanced prostate cancer. BMC Cancer. 2013;13:89.

54. Foley E, Baillie A, Huxter M, Price M, Sinclair E. Mindfulness-based cognitive therapy for individuals whose lives have been affected by cancer: a randomized controlled trial. J Consult Clin Psychol. 2010;78(1):72-9.

55. Garland SN, Tamagawa R, Todd SC, Speca $\mathrm{M}$, Carlson LE. Increased mindfulness is related to improved stress and mood following participation in a mindfulnessbased stress reduction program in individuals with cancer. Integr Cancer Ther. 2013;12(1):31-40.

56. Hoffman CJ, Ersser SJ, Hopkinson JB. Mindfulness-based stress reduction in breast cancer: a qualitative analysis. Complement Ther Clin Pract. 2012;18(4):221-6.

57. Kvillemo P, Branstrom R. Experiences of a mindfulness-based stress-reduction intervention among patients with cancer. Cancer Nurs. 2011;34(1):24-31.

58. Lengacher CA, Johnson-Mallard V, PostWhite J, Moscoso MS, Jacobsen PB, Klein TW, et al. Randomized controlled trial of mindfulness-based stress reduction (MBSR) for survivors of breast cancer. Psycho-oncology. 2009;18(12):1261-72.

59. Monti DA, Kash KM, Kunkel EJ, Moss A, Mathews M, Brainard $G$, et al. 
Psychosocial benefits of a novel mindfulness intervention versus standard support in distressed women with breast cancer. Psycho-oncology. 2013;22(11):2565-75.

60. Stafford L, Foley E, Judd F, Gibson P, Kiropoulos L, Couper J. Mindfulnessbased cognitive group therapy for women with breast and gynecologic cancer: a pilot study to determine effectiveness and feasibility. Support Care Cancer. 2013;21(11):3009-19.

61. van der Lee ML, Garssen B. Mindfulnessbased cognitive therapy reduces chronic cancer-related fatigue: a treatment study. Psycho-oncology. 2012;21(3):264-72.

62. Wurtzen H, Dalton SO, Elsass P, Sumbundu $A D$, Steding-Jensen M, Karlsen RV, et al. Mindfulness significantly reduces selfreported levels of anxiety and depression: results of a randomised controlled trial among 336 Danish women treated for stage I-III breast cancer. Eur J Cancer. 2013;49(6):1365-73.

63. Zernicke KA, Campbell TS, Speca M, McCabe-Ruff K, Flowers S, Dirkse DA, et al. The eCALM Trial-eTherapy for cancer appLying mindfulness: online mindfulnessbased cancer recovery program for underserved individuals living with cancer in Alberta: protocol development for a randomized wait-list controlled clinical trial. BMC Complement Altern Med. 2013;13:34.

64. Fox SD, Flynn E, Allen RH. Mindfulness meditation for women with chronic pelvic pain: a pilot study. J Reprod Med. 2011;56(3-4):158-62.

65. Waters AJ, Reitzel LR, Cinciripini P, Li Y, Marcus MT, Vidrine JI, et al. Associations between mindfulness and implicit cognition and self-reported affect. Subst Abus. 2009;30(4):328-37.

66. Branstrom R, Kvillemo P, Akerstedt T. Effects of mindfulness training on levels of cortisol in cancer patients. Psychosomatics. 2013;54(2):158-64.

67. Lipschitz DL, Kuhn R, Kinney AY, Donaldson GW, Nakamura Y.
Reduction in salivary alpha-amylase levels following a mind-body intervention in cancer survivors--an exploratory study. Psychoneuroendocrinology.

2013;38(9):1521-31.

68. Kocovski NL, Fleming JE, Hawley LL, Huta V, Antony MM. Mindfulness and acceptance-based group therapy versus traditional cognitive behavioral group therapy for social anxiety disorder: a randomized controlled trial. Behav Res Ther. 2013;51(12):889-98.

69. Marsland AL, Long KA, Howe C, Thompson AL, Tersak J, Ewing LJ. A pilot trial of a stress management intervention for primary caregivers of children newly diagnosed with cancer: preliminary evidence that perceived social support moderates the psychosocial benefit of intervention. J Pediatr Psychol. 2013;38(4):449-61.

70. Gielissen MF, Prins JB, Knoop H, Verhagen S, Bleijenberg G. Pictorial Representation of Self and Illness Measure (PRISM): a graphic instrument to assess suffering in fatigued cancer survivors. Psychol Assess. 2013;25(2):658-63.

71. Barth J, Delfino S, Kunzler A. Naturalistic study on the effectiveness of psycho-oncological interventions in cancer patients and their partners. Support Care Cancer. 2013;21(6):1587-95.

72. Dyer J, Thomas K, Sandsund C, Shaw C. Is reflexology as effective as aromatherapy massage for symptom relief in an adult outpatient oncology population? Complement Ther Clin Pract. 2013;19(3):139-46.

73. Prinsen $\mathrm{H}$, Bleijenberg $\mathrm{G}$, Heijmen $\mathrm{L}$, Zwarts MJ, Leer JW, Heerschap A, et al. The role of physical activity and physical fitness in postcancer fatigue: a randomized controlled trial. Support Care Cancer. 2013;21(8):2279-88.

74. Hofmann J, Peters S, Geidl W, Hentschke $\mathrm{C}$, Pfeifer K. Effects of behavioural exercise therapy on the effectiveness of a multidisciplinary rehabilitation for chronic non-specific low back pain: study protocol 
for a randomised controlled trial. BMC Musculoskelet Disord. 2013;14:89.

75. Gielissen MF, Wiborg JF, Verhagen CA, Knoop H, Bleijenberg G. Examining the role of physical activity in reducing postcancer fatigue. Support Care Cancer. 2012;20(7):1441-7.

76. Kovacic T, Zagoricnik M, Kovacic M. Impact of relaxation training according to the Yoga In Daily Life(R) system on anxiety after breast cancer surgery. J Complement Integr Med. 2013;10.

77. Isa MR, Moy FM, Razack AH, Zainuddin ZM, Zainal NZ. Impact of applied progressive deep muscle relaxation training on the health related quality of life among prostate cancer patients--a quasi experimental trial. Prev Med. 2013;57 Suppl:S37-40.

78. Suh EE. The effects of P6 acupressure and nurse-provided counseling on chemotherapy-induced nausea and vomiting in patients with breast cancer. Oncol Nurs Forum. 2012;39(1):E1-9.

79. Johnston MF, Hays RD, Subramanian SK, Elashoff RM, Axe EK, Li JJ, et al. Patient education integrated with acupuncture for relief of cancer-related fatigue randomized controlled feasibility study. BMC Complement Altern Med. 2011;11:49.

80. Chen YL, Francis AJ. Relaxation and imagery for chronic, nonmalignant pain: effects on pain symptoms, quality of life, and mental health. Pain Manag Nurs. 2010;11(3):159-68.

81. Beard C, Stason WB, Wang Q, Manola J, Dean-Clower E, Dusek JA, et al. Effects of complementary therapies on clinical outcomes in patients being treated with radiation therapy for prostate cancer. Cancer. 2011;117(1):96-102.

82. Sudarshan M, Petrucci A, Dumitra S, Duplisea J, Wexler S, Meterissian S. Yoga therapy for breast cancer patients: a prospective cohort study. Complement Ther Clin Pract. 2013;19(4):227-9.

83. Dhruva A, Miaskowski C, Abrams D, Acree M, Cooper B, Goodman
$\mathrm{S}$, et al. Yoga breathing for cancer chemotherapy-associated symptoms and quality of life: results of a pilot randomized controlled trial. J Altern Complement Med. 2012;18(5):473-9.

84. Kovacic T, Kovacic M. Impact of relaxation training according to Yoga In Daily Life(R) system on self-esteem after breast cancer surgery. J Altern Complement Med. 2011;17(12):1157-64.

85. Loudon A, Barnett $\mathrm{T}$, Piller $\mathrm{N}$, Immink MA, Visentin D, Williams AD. The effect of yoga on women with secondary arm lymphoedema from breast cancer treatment. BMC Complement Altern Med. 2012;12:66.

86. Mustian KM, Sprod LK, Janelsins M, Peppone LJ, Palesh OG, Chandwani $\mathrm{K}$, et al. Multicenter, randomized controlled trial of yoga for sleep quality among cancer survivors. J Clin Oncol. 2013;31(26):3233-41.

87. Ulger O, Yagli NV. Effects of yoga on the quality of life in cancer patients. Complement Ther Clin Pract. 2010;16(2):60-3.

88. Yadav RK, Magan D, Mehta N, Sharma R, Mahapatra SC. Efficacy of a shortterm yoga-based lifestyle intervention in reducing stress and inflammation: preliminary results. J Altern Complement Med. 2012;18(7):662-7.

89. Hendricks-Ferguson VL, Cherven BO, Burns DS, Docherty SL, Phillips-Salimi CR, Roll $\mathrm{L}$, et al. Recruitment strategies and rates of a multi-site behavioral intervention for adolescents and young adults with cancer. J Pediatr Health Care. 2013;27(6):434-42.

90. Bjorneklett HG, Lindemalm C, Ojutkangas ML, Berglund A, Letocha H, Strang P, et al. A randomized controlled trial of a support group intervention on the quality of life and fatigue in women after primary treatment for early breast cancer. Support Care Cancer. 2012;20(12):3325-34.

91. Gutgsell KJ, Schluchter M, Margevicius S, DeGolia PA, McLaughlin B, Harris $\mathrm{M}$, et al. Music therapy reduces pain 
in palliative care patients: a randomized controlled trial. J Pain Symptom Manage. 2013;45(5):822-31.

92. Madson AT, Silverman MJ. The effect of music therapy on relaxation, anxiety, pain perception, and nausea in adult solid organ transplant patients. J Music Ther. 2010;47(3):220-32.

93. Lechtzin N, Busse AM, Smith MT, Grossman S, Nesbit S, Diette GB. A randomized trial of nature scenery and sounds versus urban scenery and sounds to reduce pain in adults undergoing bone marrow aspirate and biopsy. J Altern Complement Med. 2010;16(9):965-72.

94. Good M, Albert JM, Anderson GC, Wotman S, Cong X, Lane D, et al. Supplementing relaxation and music for pain after surgery. Nurs Res. 2010;59(4):259-69.

95. Hsiao FH, Jow GM, Kuo WH, Chang KJ, Liu YF, Ho RT, et al. The effects of psychotherapy on psychological wellbeing and diurnal cortisol patterns in breast cancer survivors. Psychother Psychosom. 2012;81(3):173-82.

96. Zwerenz R, Beutel ME, Imruck BH, Wiltink J, Haselbacher A, Ruckes C, et al. Efficacy of psychodynamic short-term psychotherapy for depressed breast cancer patients: study protocol for a randomized controlled trial. BMC Cancer. 2012;12:578.

97. Jensen MP, Ehde DM, Gertz KJ, Stoelb BL, Dillworth TM, Hirsh AT, et al. Effects of self-hypnosis training and cognitive restructuring on daily pain intensity and catastrophizing in individuals with multiple sclerosis and chronic pain. Int J Clin Exp Hypn. 2011;59(1):45-63.

98. Montgomery GH, David D, Kangas M, Green S, Sucala M, et al. Randomized controlled trial of a cognitive-behavioral therapy plus hypnosis intervention to control fatigue in patients undergoing radiotherapy for breast cancer. J Clin Oncol. 2014 Feb 20;32(6):557-63. 